\title{
2019 Reviewer Acknowledgement
}

\author{
Editorial Office of Journal of Clinical and Translational Hepatology
}

We thank the following reviewers for their contribution and support in 2019.

Mai Ali Abd el Meguid

Egypt

Mohamed Abdallah Al Hajj

Abdallah

United States

Sherief Abd-Elsalam

Egypt

Anant Agarwalla

United States

Avin Aggarwal

United States

Kawtar Alkhalloufi

United States

Ahmed M. Alkhunaizi

Saudi Arabia

Khaled Amer

Egypt

Masahiro Arai

Japan

Leon Averbukh

United States

Timothy A. Bach

United States

Gyorgy Baffy

United States

Adriana de Los Anteles Barrios

Guatemala

Roopjeet Bath

United States

Ines Bilic-Curcic

CROATIA

Thomas Graham Bird

United Kingdom

John W Birk

United States

DOI: $10.14218 /$ JCTH.2019.000RA

\author{
Peter Buch \\ United States \\ Chalermrat Bunchorntavakul
Thailand \\ Ozge Telci Caklili
Turkey \\ Wenqing (Wendy) Cao \\ United States \\ Vincenzo Cardinale \\ Italy \\ Guido Carpino \\ Italy \\ Ryan Chadha \\ United States \\ Naichaya Chamroonkul \\ Thailand \\ Jason Pik Eu Chang \\ Singapore
}

Phunchai Charatcharoenwitthaya

Thailand

Limin Chen

Canada

Yongpeng Chen

China

José Artur Chies

Brazil

Ashok Choudhury

India

Alessandro Csermely

Italy

Jessica Dahmus

United States

Victor David w

United States

Giovan Giuseppe Di Costanzo Italy

Iliana B Doycheva

United States

\author{
Xingrong Du \\ United States \\ Atanu Kumar Dutta
India \\ Mohamed A. El-Guindi \\ Egypt \\ Gülsüm Ozlem Elpek \\ Turkey \\ Saleh Elwir \\ United States \\ Yu-Chen Fan \\ China \\ Jian-Gao Fan \\ China \\ Myriam Farah \\ Canada
}

Artin Galoosian

United States

Yanhang Gao

China

Antonio Giorgio

Italy

Amit Goel

India

George Boon-Bee Goh

Singapore

Humberto C Gonzalez

United States

\section{Kazuhiko Hayashi}

Japan

Noha Helal

Egypt

Jing Jing

China

sandeep kumar karn

China

Ahyoung Kim

United States 
2019 Reviewer Acknowledgement

\begin{tabular}{|c|c|c|}
\hline $\begin{array}{l}\text { John Koskinas } \\
\text { Greece }\end{array}$ & $\begin{array}{l}\text { Jie Peng } \\
\text { China }\end{array}$ & $\begin{array}{l}\text { Hsin-Yun Sun } \\
\text { Taiwan }\end{array}$ \\
\hline $\begin{array}{l}\text { Karen Krok } \\
\text { United States }\end{array}$ & $\begin{array}{l}\text { Cyriac Abby Philips } \\
\text { India }\end{array}$ & $\begin{array}{l}\text { Sridhar Sundaram } \\
\text { India }\end{array}$ \\
\hline $\begin{array}{l}\text { Pramod Kumar } \\
\text { India }\end{array}$ & $\begin{array}{l}\text { Madhumita Premkumar } \\
\text { India }\end{array}$ & $\begin{array}{l}\text { Ashraf Tabll } \\
\text { Egypt }\end{array}$ \\
\hline $\begin{array}{l}\text { Ewa Kurys-Denis } \\
\text { Poland }\end{array}$ & $\begin{array}{l}\text { Xingshun Qi } \\
\text { China }\end{array}$ & $\begin{array}{l}\text { Tawesak Tanwandee } \\
\text { Thailand }\end{array}$ \\
\hline $\begin{array}{l}\text { Chaoshuang Lin } \\
\text { China }\end{array}$ & $\begin{array}{l}\text { Bo Qin } \\
\text { China }\end{array}$ & $\begin{array}{l}\text { Georgios Tsoulfas } \\
\text { Greece }\end{array}$ \\
\hline $\begin{array}{l}\text { Cheng-Hai Liu } \\
\text { China }\end{array}$ & $\begin{array}{l}\text { Atoosa Rabiee } \\
\text { United States }\end{array}$ & $\begin{array}{l}\text { Lucija Virovic-Jukic } \\
\text { Croatia }\end{array}$ \\
\hline $\begin{array}{l}\text { Xiaocong Liu } \\
\text { United States }\end{array}$ & $\begin{array}{l}\text { Chitta Ranjan } \\
\text { India }\end{array}$ & $\begin{array}{l}\text { Benjamin I Woolbright } \\
\text { United States }\end{array}$ \\
\hline $\begin{array}{l}\text { Shousheng Liu } \\
\text { China }\end{array}$ & $\begin{array}{l}\text { Sahaj Rathi } \\
\text { Canada }\end{array}$ & $\begin{array}{l}\text { QIULIXIE } \\
\text { China }\end{array}$ \\
\hline $\begin{array}{l}\text { Joyce A. Lloyd } \\
\text { United States }\end{array}$ & $\begin{array}{l}\text { Aathira Ravindranath } \\
\text { India }\end{array}$ & $\begin{array}{l}\text { Chencheng Xie } \\
\text { United States }\end{array}$ \\
\hline $\begin{array}{l}\text { Lungen Lu } \\
\text { China }\end{array}$ & $\begin{array}{l}\text { Ciro Romano } \\
\text { Italy }\end{array}$ & $\begin{array}{l}\text { Yongning Xin } \\
\text { China }\end{array}$ \\
\hline $\begin{array}{l}\text { Mojtaba mafi } \\
\text { Iran (Islamic Republic of) }\end{array}$ & $\begin{array}{l}\text { Farzin Roohvand } \\
\text { Iran (Islamic Republic of) }\end{array}$ & $\begin{array}{l}\text { Weijie Xu } \\
\text { China }\end{array}$ \\
\hline $\begin{array}{l}\text { Sunil K Malonia } \\
\text { United States }\end{array}$ & $\begin{array}{l}\text { Mehdi Sadat } \\
\text { Iran (Islamic Republic of) }\end{array}$ & $\begin{array}{l}\text { Po-Chih Yang } \\
\text { Taiwan }\end{array}$ \\
\hline $\begin{array}{l}\text { Alessandro Mantovani } \\
\text { Italy }\end{array}$ & $\begin{array}{l}\text { Ghada Salum } \\
\text { Egypt }\end{array}$ & $\begin{array}{l}\text { Eric M Yoshida } \\
\text { Canada }\end{array}$ \\
\hline $\begin{array}{l}\text { Qing Mao } \\
\text { China }\end{array}$ & $\begin{array}{l}\text { Keisaku Sato } \\
\text { United States }\end{array}$ & $\begin{array}{l}\text { Yuexin Zhang } \\
\text { China }\end{array}$ \\
\hline $\begin{array}{l}\text { Rosa Maria Martin-Mateos } \\
\text { Spain }\end{array}$ & $\begin{array}{l}\text { Moinak Sen Sarma } \\
\text { India }\end{array}$ & $\begin{array}{l}\text { Rong Zhang } \\
\text { United States }\end{array}$ \\
\hline $\begin{array}{l}\text { Yong-Ping Mu } \\
\text { China }\end{array}$ & $\begin{array}{l}\text { Ke-Qing Shi } \\
\text { China }\end{array}$ & $\begin{array}{l}\text { Qingzhan Zhang } \\
\text { United States }\end{array}$ \\
\hline $\begin{array}{l}\text { Seyedehsan Navabi } \\
\text { United States }\end{array}$ & $\begin{array}{l}\text { Hao Shi } \\
\text { United States }\end{array}$ & $\begin{array}{l}\text { Ze-Hua Zhao } \\
\text { China }\end{array}$ \\
\hline $\begin{array}{l}\text { Paul Naylor } \\
\text { United States }\end{array}$ & $\begin{array}{l}\text { Coleman Smith } \\
\text { United States }\end{array}$ & $\begin{array}{l}\text { Ming-Hua Zheng } \\
\text { China }\end{array}$ \\
\hline $\begin{array}{l}\text { Bolin Niu } \\
\text { United States }\end{array}$ & $\begin{array}{l}\text { Bin Song } \\
\text { China }\end{array}$ & $\begin{array}{l}\text { Yueyong Zhu } \\
\text { China }\end{array}$ \\
\hline $\begin{array}{l}\text { James Park } \\
\text { United States }\end{array}$ & $\begin{array}{l}\text { Hang Sun } \\
\text { China }\end{array}$ & $\begin{array}{l}\text { Julie Zhu } \\
\text { Canada }\end{array}$ \\
\hline
\end{tabular}

